\title{
An automatic constructive matheuristic for the shift minimization personnel task scheduling problem
}

\author{
Reshma Chirayil Chandrasekharan . \\ Pieter Smet . Tony Wauters
}

Received: date / Accepted: date

\begin{abstract}
The shift minimization personnel task scheduling problem is an NP-complete optimization problem that concerns the assignment of tasks to multi-skilled employees with a view to minimize the total number of assigned employees. Recent literature indicates that hybrid methods which combine exact and heuristic techniques such as matheuristics are efficient as regards to generating high quality solutions. The present work employs a constructive matheuristic $(\mathrm{CMH})$ : a decomposition-based method where sub-problems are solved to optimality using exact techniques. The optimal solutions of subproblems are subsequently utilized to construct a feasible solution for the entire problem. Based on the study, a time-based $\mathrm{CMH}$ has been developed which, for the first time, solves all the difficult instances introduced by Smet et al. (2014) to optimality. In addition, an automated CMH algorithm that utilizes instance-specific problem features has also been developed that produces high quality solutions over all current benchmark instances.
\end{abstract}

Keywords Task Scheduling · decomposition · SMPTSP · matheuristic

Reshma Chirayil Chandrasekharan

KU Leuven, Department of Computer Science, CODeS, Gebroeders De Smetstraat 1, 9000 Gent, Belgium

E-mail: ReshmaCC@kuleuven.be

Pieter Smet

KU Leuven, Department of Computer Science, CODeS, Gebroeders De Smetstraat 1, 9000 Gent, Belgium

E-mail: pieter.smet@kuleuven.be

Tony Wauters

KU Leuven, Department of Computer Science, CODeS, Gebroeders De Smetstraat 1, 9000

Gent, Belgium

E-mail: tony.wauters@kuleuven.be 


\section{Introduction}

Personnel scheduling concerns assigning tasks to a given workforce such that a certain cost is minimized. Due to its widespread applications and connection to other optimization problems, personnel scheduling continues to constitute one of the most active research areas within operations research (den Bergh et al., 2013). The influence and applicability of other research streams such as behavioural sciences and human rights also serves to maintain the particularly interesting status of personnel scheduling.

The problem of determining the size and composition of a minimal workforce required to perform a specific operation is encountered often in personnel scheduling. It becomes even more difficult when there is a multi-skilled workforce of which only some are skilled to perform certain tasks. This problem was studied by Dowling et al. (1997) to develop a day-to-day planning tool and to estimate a minimal staff set capable of operating as the ground staff of an international airport. The same problem can also be viewed as a machine scheduling problem if shifts are mapped to machines and tasks to jobs (Kolen et al., 2007). Another way of looking at the problem is in the form of a colouring problem on the interval graph defined by the time intervals associated with tasks. Motivated by its applicability in personnel rostering, Krishnamoorthy et al. (2012) formally defined the problem and introduced it as a variant of the personnel task scheduling problem. A benchmark dataset of 137 instance was proposed and two heuristics were utilized to generate 135 feasible solutions of which 70 were optimal. To enable this dataset to also be applicable for machine scheduling, it is assumed that shifts/employees are available throughout the planning horizon $(24 \mathrm{hrs})$. In personnel scheduling, these long shifts can be subdivided and performed by one or more personnel with similar skills. The problem is therefore equivalent to determining the minimal set of shifts required and is called the shift minimization personnel task scheduling problem (SMPTSP). For the ease of discussion, the SMPTSP will be presented as a problem concerning assignment of employees to tasks similar to how it has been treated in every other paper in the literature.

This problem is proven NP-Complete and therefore, with the exception of very small instances involving only a few tasks and shifts, exact approaches turn out to be inefficient at generating high quality solutions. As a consequence, most of the solution approaches found in the literature are heuristic in nature. Lin and Ying (2014) introduced a three-phase heuristic approach which was able to generate 135 feasible solutions, of which 101 were optimal. Their approach involved a constructive heuristic and an iterated greedy heuristic, with the generated solutions utilized by the general purpose solver Gurobi. Employing a multi-stage approach, Smet et al. (2014) was successful in solving all 137 instances to optimality for the first time. In addition to their promising results, a major contribution of the paper was the proposal of a constructive matheuristic $(\mathrm{CMH})$ approach. The authors also conducted a detailed study of the benchmark instances and a new benchmark dataset of ten difficult instances was proposed, with their approach being capable of solving 
five of these to optimality. Since then, few methods have been proposed in an attempt to solve the remaining unsolved instances to optimality. Baatar et al. (2015) introduced a novel branching scheme based on the mathematical structure of the problem and applied two column generation methods and a heuristic. Their approach was able to solve 6 out of these 10 instances to optimality. An optimality-preserving decomposition method was discussed in Niraj Ramesh et al. (2018) which was capable of solving 5 of them and 83 of the 137 original benchmark instances to optimality. Four of these ten difficult instances therefore, still remain unsolved. In addition, a new benchmark dataset with different structure was introduced by Fages and Lapègue (2013) and their approach solved 46 of them to optimality. Later, 84 of them were solved by Hojati (2018).

The aim of this study is twofold. Existing methods in the literature are not efficient at producing optimal solutions for all benchmark instance sets. The first aim of this study therefore is to develop a solution approach that takes instance structure into account to produce high quality solutions for all sets of instances. Second, since previous research such as Smet et al. (2014) has motivated the implementation of a $\mathrm{CMH}$ approach so that large real world scenarios can also be solved effectively, is to investigate the applicability of $\mathrm{CMH}$ techniques for the SMPTSP and to test additional CMH-design parameters defined in Chandrasekharan et al. (2019).

To begin with, the first part of the study focuses on selecting an appropriate decomposition for the SMPTSP that is suitable to be employed in a $\mathrm{CMH}$ framework. This has led to the development of a $\mathrm{CMH}$ approach that assumes fixed values for the design parameters predefined by the user. In the second part, interaction of the design parameters with the instance structure is studied and as a result, the $\mathrm{CMH}$ approach has been extended to be able to arrive at suitable values for certain design parameters based on the instance structure. This automated heuristic approach has been able to generate high quality solutions for a large number of benchmark instances over the three different instance sets. The following section formally introduces the problem and its mathematical formulation. Section 3 introduces the overall strategy utilized in this paper and provides a comparative study concerning two possible decomposition approaches. Computational experiments are detailed in Section 5 and Section 5.2 describes the improved CMH strategy. Section 6 presents the final results and compares these against the state of the art. Finally, Section 7 summarizes the conclusions.

\section{The shift minimization personnel task scheduling problem}

The SMPTSP involves a set of tasks and employees. A task is spread over some time interval and has specification concerning the skill set required to perform it. An employee is characterised by its start and end times and has a set of tasks associated which it is skilled to perform. Tasks must be performed 
one at a time by a given employee. The aim of the SMPTSP is to determine a minimal set of employees capable of performing all tasks.

Let the set of tasks and employees be denoted by $J=\{1,2, \ldots, n\}$ and $W=\{1,2, \ldots, m\}$, respectively. Task $j$ can be performed by employee $w$ if $w$ has the skills required to perform task $j$ and is available during the time interval associated with task $j$. Let the set of employees that can perform task $j$ be denoted by $W_{j} \subseteq W$. Similarly, for every employee $w$, let $J_{w} \subseteq J$ be the set of tasks which $w$ can perform. Note that two tasks which overlap in time cannot be assigned to the same employee.

Consider graph $G$ defined with respect to the set of tasks $J$. Two tasks in $G$ are connected if they overlap in time. Note that any two tasks in a maximal clique of $G$ overlap in time and therefore cannot be assigned to the same employee. For each employee $w$, consider the induced subgraph $G_{w}$ on the vertices from set $J_{w}$. Let $C^{w}=\left\{K_{1}, K_{2}, . ., K_{t}\right\}$ be the set of maximal cliques in $G$. Note that no two tasks in $K_{l} \in C^{w}$ can be assigned to the same $w$.

The objective of the SMPTSP is to find a minimal subset of $W$ that is capable of performing all the tasks in $J$. In this paper, the Intger Programming (IP) formulation introduced by Krishnamoorthy et al. (2012) is utilized, which makes use of the following two sets of decision variables:

$$
\begin{aligned}
x_{j w} & = \begin{cases}1 & \text { if task } j \in J \text { is assigned to employee } w \in W \\
0 & \text { otherwise }\end{cases} \\
y_{w} & = \begin{cases}1 & \text { if employee } w \in W \text { is active } \\
0 & \text { otherwise }\end{cases}
\end{aligned}
$$

The model can then be formulated as:

$$
\begin{aligned}
& \text { minimize: } \sum_{w \in W} y_{w} \\
& \text { subject to: } \quad \sum_{w \in W_{j}} x_{j w}=1 \quad \forall j \in J \\
& \sum_{j \in K_{l}^{w}} x_{j w} \leq y_{w} \quad \forall w \in W, \forall K_{l} \in C^{w} \\
& 0 \leq y_{w} \leq 1 \quad \forall w \in W \\
& x_{j w} \in\{0,1\} \quad \forall j \in J, \forall w \in W
\end{aligned}
$$

The objective of the SMPTSP is provided by way of Equation (3) and seeks to minimize the number of employees utilized. Constraints (4) ensure that a task is assigned to exactly one employee. Constraints (5) stipulate that for any employee $w$, no two tasks in a maximal clique of $G_{w}$ are assigned to it and that tasks are assigned only to active employees. Constraints (6) and (7) specify bounds of the decision variables. 


\subsection{Benchmark data set}

Krishnamoorthy et al. (2012) proposed the dataset comprising of 137 instances, denoted by Data_137. The parameters associated with each instance are its number of employees, task lengths, multi-skilling level of employees and the tightness $(T \%)$, defined as the total length of all tasks as a percentage of the total duration of employees. Time interval for each instance was 24 hours (1440 mins). Therefore, starting time of the instance $t_{\min }$ and its end time $t_{\max }$ are fixed as 0 and 1440 respectively. Smet et al. (2014) proved that the size of largest maximal clique in graph $G$ is a valid lower bound for the SMPTSP, referring to it as the clique lower bound (CLB). The same properties also hold for the 10 difficult instances proposed, denoted by Data_10, as well. However, for the 100 instances proposed by Fages and Lapègue (2013), denoted by Data_100, CLBs not necessarily correspond to optimal solutions. Following this work, Solyali (2016) proposed a general lower bounding procedure which established efficient lower bounds for these instances.

\section{Constructive matheuristic}

Maniezzo et al. (2009) define matheuristics as algorithmic hybrids of mathematical programming and heuristics. Lately, various heuristics have been designed which incorporate Mixed Integer Programming (MIP) solvers, given recent advances in the area. These methods are referred to as MIP heuristics and, by definition, belong to the class of matheuristic algorithms. The local branching technique presented in Fischetti and Lodi (2003), which iteratively solves subsets of variables mathematically and fix them, is an example of one such heuristic. Other successful examples of MIP heuristics include heuristics utilized in Santos et al. (2016) and Della Croce et al. (2014).

Smet et al. (2014) introduced a simple MIP heuristic which sequentially solves subproblems using a commercial MIP solver, thereby arriving at a feasible solution for the entire problem. The generality of the $\mathrm{CMH}$ strategy presented in Smet et al. (2014) makes it easily adaptable and therefore is utilized by the present paper. Two different decompositions are employed in this framework and additional design parameters defined in Chandrasekharan et al. (2019) are tested to arrive at conclusions regarding the interaction of problem structure and the two decompositions. Much like any other decompositionbased method, the challenge is to identify an appropriate decomposition strategy which results in high quality solutions. Once a decomposition approach has been determined, an order for solving these subproblems is determined and they are then solved one by one. It is often the case that not all hard constraints can be evaluated locally within individual subproblems. Such constraints are therefore converted into appropriate soft constraints and employed in the subproblems such that their feasibility is maintained in the final solution produced by the $\mathrm{CMH}$. The decomposition strategy, the sub problems' 
formulation and order of execution of these subproblems, all constitute key factors that define a CMH strategy.

\subsection{The general CMH framework for the SMPTSP}

A given problem is decomposed into subproblems referred to as blocks. An IP formulation for each block is solved using a generic MIP solver as it is encountered. The values of a block's decision variables are then fixed. The CMH continues solving blocks until either the final block is solved or an infeasibility is encountered. The following design parameters are utilized.

1. Block size $(\eta)$ : Subproblem size is central in determining the solution quality and CMH runtimes. While larger subproblems are typically associated with better solutions, they also result in longer run times. The size of blocks must therefore be carefully chosen in order to define an effective decomposition strategy.

2. Overlap $(\theta)$ : A challenge when defining subproblems is that the fixed values of previously-solved blocks affect the feasibility and solution quality of the blocks yet to be solved. The CMH permits overlap between subproblems so that the feasibility of the subproblems yet to be solved is taken into some degree of consideration while solving the current one. This design parameter defines the extent of overlap between subproblems.

3. Relaxed future $(\rho)$ : While larger subproblems often lead to better solutions, increasing subproblem size is not always feasible due to runtime restrictions. This parameter extends a subproblem by including a part of the next subproblem in a relaxed manner. This relaxed portion of the next subproblem is named relaxed future and the parameter $\rho$ determines its size. In problems where there are hard constraints spanning across different blocks and for which applying a relax and fix method (Wolsey, 1998) might not be feasible due to long root relaxation solve times, incorporating a large relaxed future can be helpful. Note that $\rho$ must be set such that the relaxed future of the current block is re-solved in the following blocks with the original formulation in order to maintain feasibility.

The CMH parameter configuration is thus given by a tuple of the format $(\eta, \theta, \rho)$. An overlap $\theta$ means that the current block is solved with a $\theta \%$ overlap with the next block. Similarly, relaxed future $\rho$ indicates the percentage of the future block relaxed and included in the current one.

Two decomposition strategies are presented in this section and they define subproblems or blocks as a subset of employees and tasks. The primary difference between the two strategies can be attributed to the very nature of decompositions employed: employee-based and time-based. 


\subsection{Employee-based decomposition}

This is the decomposition strategy employed in the CMH introduced by Smet et al. (2014) for the SMPTSP. In this decomposition, a block is defined by a subset of employees $W^{\prime} \subseteq W$ and the set of unassigned tasks $J$ which can be performed by $W^{\prime}$. An IP formulation of the problem is solved and the block's assignments are fixed. Since Constraints (4) cannot be evaluated on a subset of the tasks, the IP formulation for the full problem cannot be evaluated for a block. Instead, a modified version where the objective is to maximize total assigned task duration is utilized. This in turn reduces the number of employees required. The following is the modified objective function utilized by blocks.

$$
\text { maximize: } \sum_{w \in W^{\prime}} \sum_{j \in J_{w}} d_{j}
$$

where $d_{j}$ is the duration of task $j$. The last block is re-optimized with Objective (3) to minimize the number of employees. Note that in this $\mathrm{CMH}$ there can be unassigned tasks after the last block is solved. In order to remove remaining infeasibilities, Smet et al. (2014) applied a local branching-based improvement heuristic on the solutions of the $\mathrm{CMH}$.

\subsection{Time-based decomposition}

The problem can also be decomposed by way of separating the problem's timeline into intervals, each of which corresponds to a block. Associated with each block is the following: a time interval, the tasks which begin in this time interval and the set of employees qualified to perform these tasks. The original IP formulation is utilized here with the only modification being that Constraints (4) only considers tasks belonging to the current block. When a task is assigned to an employee, the time interval associated with the task is deleted from the employee's timeline in order to prevent overlapping task assignments in future blocks. A block may become infeasibile if there are no employees with the required skill set left to execute tasks which are part of it. Note, however, that in this decomposition, either a block becomes infeasible or all tasks are assigned when $\mathrm{CMH}$ completes execution.

Figure 1 presents a snapshot in time of the two different decompositions. Employees and tasks are presented as time intervals and rectangular windows represents blocks. Tasks are numbered and the list of numbers written on an employee are the tasks which it is skilled to perform. As evident from the figure, block in the employee based decomposition are defined by subsets of employees whereas blocks in the time based decomposition are time intervals.

\section{Automatic time-bsaed CMH}

For a given instance, all employees span the entire time horizon while tasks are distributed over it. Figure 2 plots the number of tasks active at each moment 


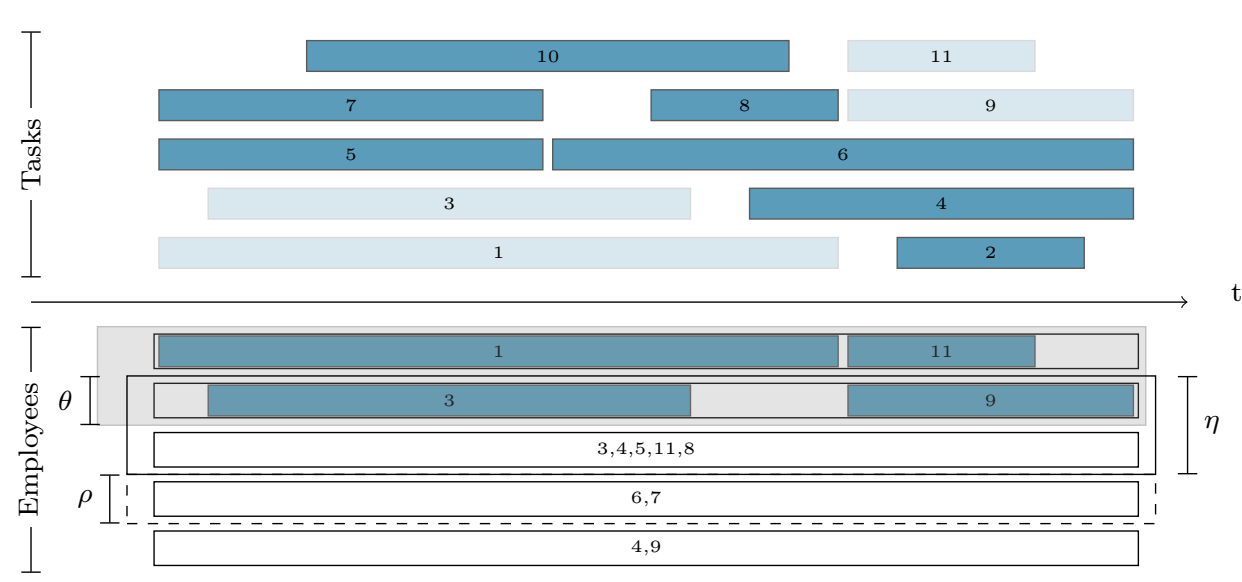

(a) Employee-based decomposition

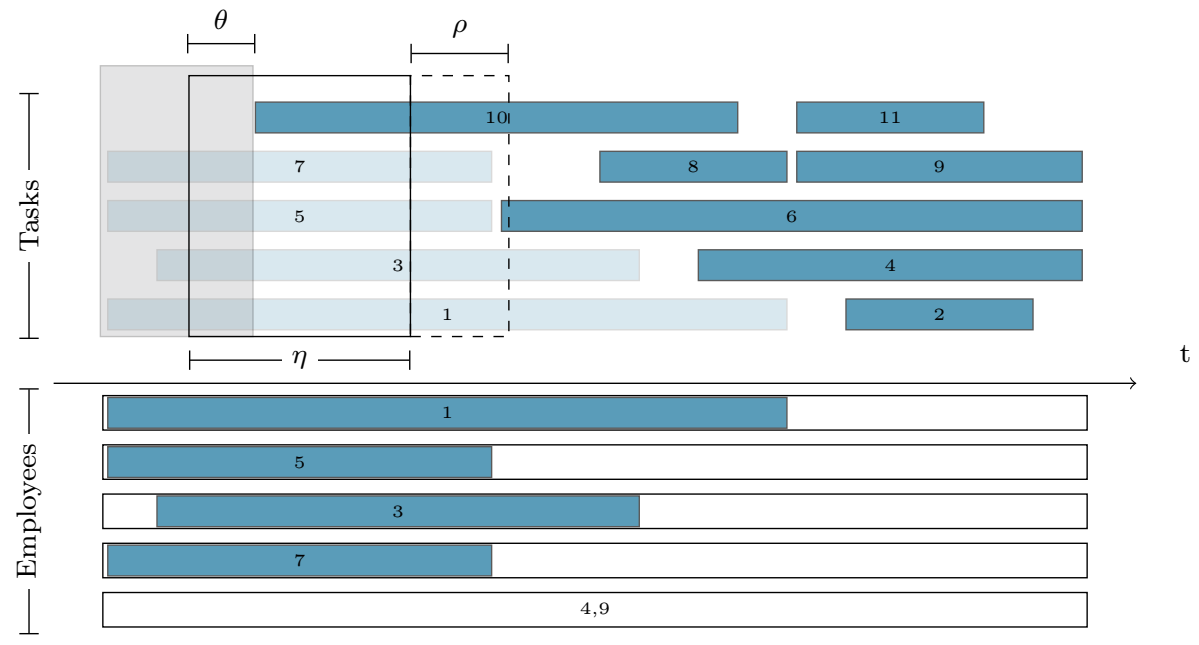

(b) Time-based decomposition

Fig. 1: A snapshot of the two CMH decompositions. The solid window is the current block, the gray window represents the previous block and the dashed window represents the relaxed future of the current block.

in time for the instances 33 and 135 of Data 100 and Data 137, respectively. The zeroes of such a graph correspond to time where no tasks are simultaneously active. Tasks active between pairs of consecutive zeroes can therefore be allotted to separate sets which can then be independently assigned to employees without compromising the optimality of the overall solution. The two graphs presented correspond to instances with contrasting properties. Instance 135 has a near-equal number of active-tasks throughout the time horizon, while the graph concerning instance 33 is characterized by values quite close to zero. Such points in time are good candidates for decomposition with respect to 
time. It has been observed that a large number of the Data 100 instances exhibit this potential for decomposability while most of the $\bar{D}$ ata_137 and Data_10 instances do not.

Instance 33 (Data 100)

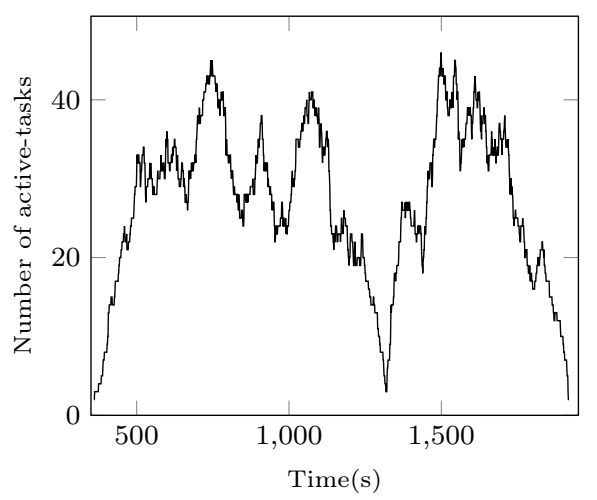

Instance 135 (Data 137)

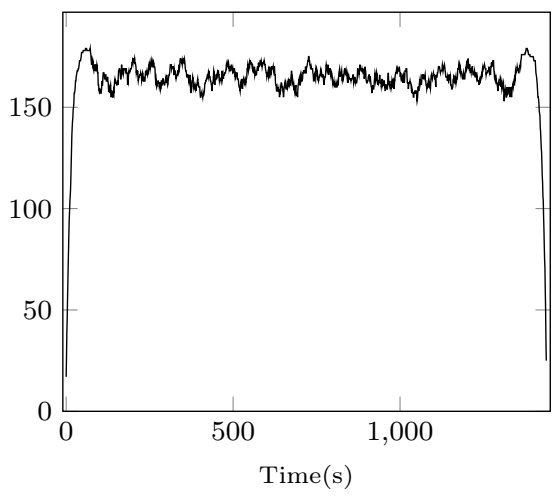

Fig. 2: The active-task distribution graph of instance 33 of Data_100 and instance 135 of Data_137

Based on the observations concerning instance structure, a strategy has been arrived at which constructs blocks based on the distribution of tasks over the time horizon. Given an instance, its active task distribution graph over time is plotted and small fluctuations are ignored. If $t_{\min }$ and $t_{\max }$ denote the start and end time values of the time horizon, the graph's set of minima in the interval $\left(t_{\text {min }}, t_{\max }\right)$, denoted by set $M=\left\{m_{1}, m_{2}, \ldots, m_{l}\right\}$, is identified. Based on $M$, the time horizon is decomposed as $\left[t_{\min }, m_{1}\right],\left[m_{1}, m_{2}\right], \ldots,\left[m_{l}, t_{\max }\right]$. Set of tasks starting in each time interval and employees skilled to perform them constitute blocks. Clearly, blocks here are of various sizes and therefore, this strategy is referred as the variable blocking strategy.

While it is possible to identify an efficient variable blocking strategy for some instances, not all can be effectively decomposed using this strategy. For instances which have a near-equal number of tasks distributed throughout the time horizon, this decomposition strategy fails at finding an effective minima for the graph (such as in instance 135 in Figure 2). It may also happen that the minima are located too close to each other such that the resulting blocks are too small to lead to high quality final solutions. An opposite scenario is when the minima are located too far from each other and, as a consequence, certain blocks are too large to be efficiently solved as a subproblem.

To be able to utilize the advantages of the variable blocking strategy while ensuring an effective decomposition, an automatic time-based CMH approach has been designed that selects between the fixed and variable blocking strategies based on instance structure. Given an instance, this approach generates 
a variable blocking strategy. If the variable blocking identified is such that all minima of the active task distribution graph are at least $r \%$ less than the maxima, the variable blocking strategy is accepted. In addition, the distance between any two consecutive minima are not allowed to be less than $d_{\min }$ in order to prevent blocks which are too small. If the approach is unable to identify a feasible variable blocking strategy, an alternative blocking strategy is applied: fixed blocking strategy. This strategy applies a simple CMH which employs the time-based decomposition. Depending on the value of $\eta$, the time horizon is decomposed into fixed equal intervals, each of which corresponds to a block.

\section{Computational study}

The CMH algorithms were coded in Java and the commercial mathematical solver Gurobi 7.0.2 was utilized to solve all subproblems. Experiments were performed on four threads of an Intel(R) Xeon(R) CPU E5-2650 v2 @ 2.60GHz computer running Ubuntu 16.04.2 LTS. The benchmark time limit is considered to be 1800 seconds. ${ }^{1}$ For the ease of discussion, the Data 137 instances are subdivided into three groups, such that instances 1-50, 51-100 and 101137 , and henceforth are referred to as small, medium and large instances, respectively.

\subsection{Comparing employee-based and time-based decompositions}

This section presents a comparative study of the employee-based and timebased decompositions when applied to the SMPTSP. The aim of the study is to test the effect of the CMH's design parameters upon final solution quality when coupled with the decompositions. Smet et al. (2014) has already tested the employee-based CMH on the Data_137 instances. This section repeats some of those experiments and extends them to the whole set of benchmark instances. Trends in design parameters are analyzed separately for Data_137 and Data_100 in order to present the variation with respect to the dataset utilized. Additional CMH design parameters are also discussed.

For a given value of $\eta$, this decomposition divides the employees into groups of size $\eta$ with each of these groups constituting a block. The order in which employees are assigned may have an impact on solution quality. Smet et al. (2014) tested the employee-based the decomposition when they were arranged in three different orders: (i) when employees which are least skilled were assigned first, (ii) when employees which are the most skilled were assigned first and (iii) when employees were randomly assigned. It was observed that these

\footnotetext{
1 The benchmark data sets Data 137, Data 10 and Data 100 are available at http://people.brunel.ac.uk/ mastjjb/jeb/info.html, https://people.cs.kuleuven. be/ pieter.smet/smptsp.html and https://sites.google.com/site/ptsplib/smptsp/ instances respectively.
} 
orders did not have a significant impact in the solution quality when the employees have a random skill structure. Therefore in the following experiments, employees are selected in a random order to construct blocks.

Figure 3 compares the solution quality and the algorithm runtime of the employee-based decomposition for block sizes $\eta=\{10,15,20\}$. This decomposition solves almost all Data_137 instances while most of the Data_100 instances remain unsolved. It is evident from the graph that larger block sizes in general lead to improved performance, but at the expense of longer run times. The employee-based decomposition executes block sizes $\eta=\{10,15,20\}$ in ascending order untill an optimal solution is found or the runtime limit is reached. As expected, the results of Data_137 results coincide with those of the same experiments presented in Smet et al. (2014). Note that the block objective function (8), which results naturally from the employee-based decomposition, does not guarantee that all tasks are assigned when the algorithm finishes execution. In other words, a feasible solution for the algorithm need not be a feasible solution for the actual problem. Smet et al. (2014) utilized a local-branching based improvement heuristic to remove infeasibilities and to improve the solution. The solutions obtained by the employee-based decomposition and the results after employing this improvement heuristic, which constitutes the current best known solutions for the SMPTSP, are summarized in Table 1 for comparison purposes. The employee-based decomposition which is capable of generating optimal solutions for most Data_137 instances is, however, not efficient in solving most of the Data_100 instances to optimality. This can be attributed to the less uniform skill structure of the Data_100 instances. While improvements might be possible by allowing multiple iterations of the algorithm with varying random employee-ordering, to accommodate instances with a non-uniform skill structure the employee-based decomposition requires determining a suitable employee-order to be utilized by the $\mathrm{CMH}$.
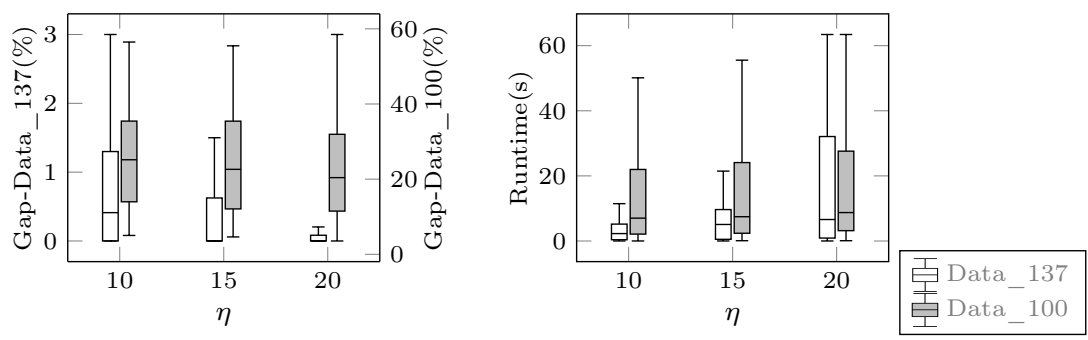

Fig. 3: Performance and algorithm runtime of the employee-based decomposition for different block sizes

Utilizing design parameters such as overlap and relaxed future has proven useful in similar CMH approaches such as Chandrasekharan et al. (2019). In an attempt to produce optimal solutions with the employee-based decomposition without the improvement heuristic and to possibly generate optimal solutions 
for the unsolved difficult Data_10 instances, overlap and relaxed future parameters were tested with respect to the employee-based decomposition. Figure 4 presents the trend of employee-based decomposition's outcome with respect to increasing overlap and relaxed future. Here, relaxed future is achieved by relaxing the integrality constraints. It is evident from the graph that neither overlap nor relaxed future brings any significant difference to solution quality. Before concluding that the overlap and relaxed future features are not beneficial for this problem, the progress of the employee-based $\mathrm{CMH}$ was observed in detail when overlap is implemented. Different employee-orders were tested to arrive at a decomposition that enables employees with tasks corresponding to overlapping time-windows to be assigned tasks simultaneously. This idea motivates the time-based decomposition in which sets of employees with the maximum number of overlapping tasks form blocks.
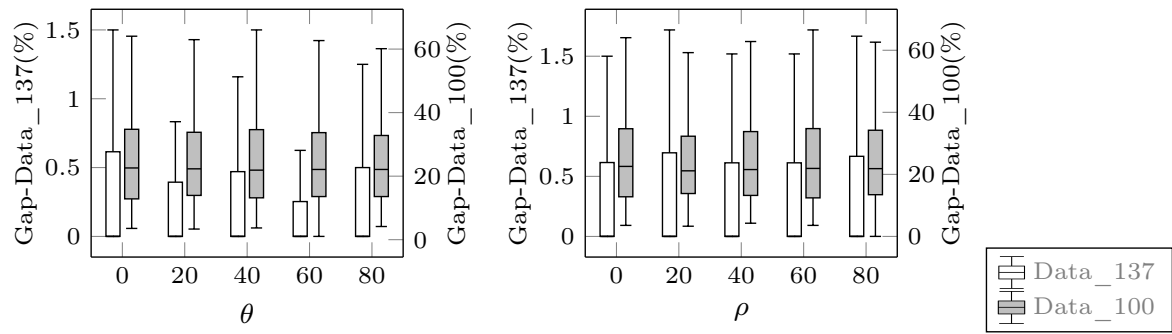

Fig. 4: Trend in the solution quality of the employee-based decomposition with respect to increasing overlap and relaxed future

In the time-based decomposition, tasks are ordered based on their start times which range from $T_{\min }$ to $T_{\max }$. Block size $\eta$ is defined as $1440 / \beta$, where $\beta$ assumes values in $\{2,3,4,5,6\}$. Once a value of $\beta$ is chosen, the timeline of the instance is subdivided into $\beta$ equal parts, each of which corresponds to a block. The block-objective is to optimally assign all the tasks within it to the set of available employees such that the total number of employees utilized is minimized. In contrast with the employee-based decomposition, a solution generated by the task-based decomposition is a feasible solution for the problem. However, it may still happen that employees are not available to perform all the tasks in a block resulting in an infeasibility. The algorithm, therefore, may fail to construct a solution. Figure 5 compares the time-based decomposition's outcome and runtime for different block sizes on data_100 and Data_137 instances when implemented with no overlap or relaxed future. It is evident from the graphs that the solutions improve with increasing block size, and so do the runtimes. In contrast with the employee-based decomposition, the time-based decomposition exhibits similar trend in both the datasets. It is also worth noting that for many large instances, the run time exceeds benchmark time limits for the largest block size corresponding to $\beta=2$. Such data points are outliers and therefore are not visible in the graphs. 

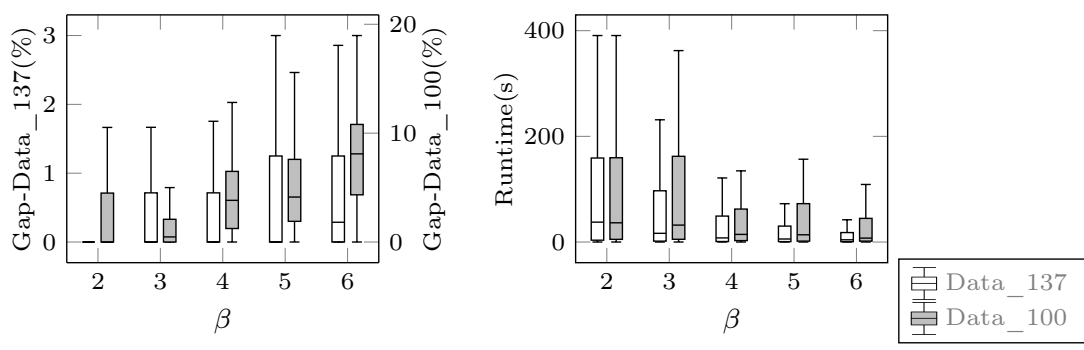

Fig. 5: Performance and algorithm runtime of time-based decomposition for different block sizes

Similar overlap and relaxed future experiments were also conducted for the time-based $\mathrm{CMH}$, with Figure 6 comparing the time-based CMH's solution quality when overlap and relaxed future are added. Although the trends are not monotonic, this plot clearly shows how overlap and relaxed future parameters can be utilized to bring significant solution quality improvements.
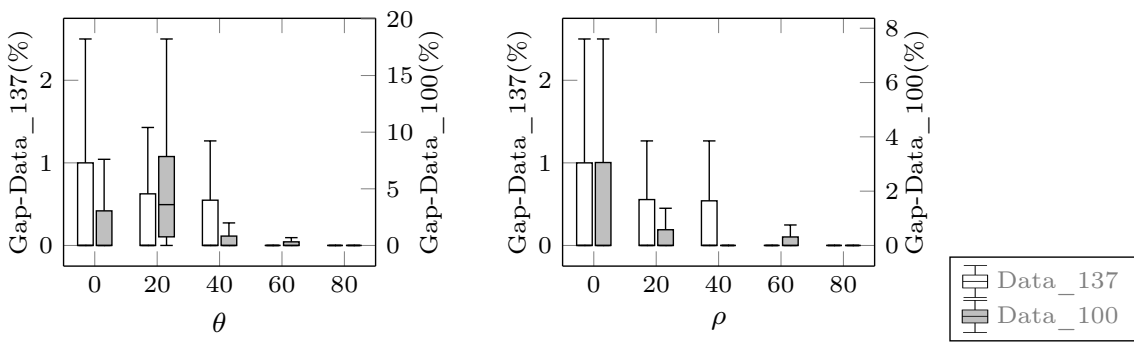

Fig. 6: Trend in the solution quality of time-based decomposition with respect to increasing overlap and relaxed future

A time-based decomposition approach was developed, which executes the configurations $(\beta, 80,0),(\beta, 90,0),(\beta, 80,50)$ and $(\beta, 90,50)$ one after the other. The values of $\theta$ and $\rho$ are selected based on the analysis from Figure 6. While larger values of $\rho$ may improve the solution quality, runtime restrictions do not support this. For each of the configurations, all values of $\beta(2,3,4,5$ and 6$)$ are tested in the descending order. The algorithm terminates when the runtime limit is reached, when an optimal solution is found or when all configurations have been executed. Table 1 and 2 summarize the performance details of this algorithm on the benchmark dataset. For the first time, all Data_10 instances have been solved to optimality. Moreover, all Data_137 instances have also been solved to optimality. The Data_100 instances, however, have not all been solved to optimality using this time-based decomposition. A closer look at the results of the Data 100 instances reveal that most of the unsolved instances are those which include very large numbers of staff $(m>500)$. Concerning the 
Data_100 instances, there certainly exists general trend upwards in solution quality with respect to increasing block size, overlap and relaxed future parameters, but one which fluctuates with some irregularity. In many Data_100 instances, larger block sizes produced optimal solutions much faster than when utilizing a smaller block size. In order to investigate this, the active task distribution in the instance-timeline was studied, which led to the development of an automatic time-based $\mathrm{CMH}$.

Table 1: Results of time-based decomposition (TBD), employee-based decomposition (EBD) and the current best known results of Smet et al. (2014)(SWMV).

\begin{tabular}{|c|c|c|c|c|c|c|c|c|c|}
\hline \multirow[b]{2}{*}{ Dataset } & \multicolumn{3}{|r|}{ EBD } & \multicolumn{3}{|r|}{ SWMV } & \multicolumn{3}{|r|}{ TBD } \\
\hline & 137 & 100 & 10 & 137 & 100 & 10 & 137 & 100 & 10 \\
\hline No of optimal solutions & 133 & 28 & 0 & 137 & 98 & 5 & 137 & 77 & 10 \\
\hline Average calculation time(s) & 44.62 & 6.4 & 19.17 & 55 & 88.46 & $1,294.15$ & 48.25 & 746.97 & 162.59 \\
\hline
\end{tabular}

Table 2: Comparison of results of time-based decomposition (TBD), employeebased decomposition (EBD) and Smet et al. (2014)(SWMV) on Data 10 instances. LB, Obj. and T(s) denote lower bound, objective-value and algorithm runtime (seconds) respectively.

\begin{tabular}{|c|c|c|c|c|c|c|c|}
\hline \multirow[b]{2}{*}{ Instances } & \multirow[b]{2}{*}{ LB } & \multicolumn{2}{|r|}{ EBD } & \multicolumn{2}{|r|}{ SWMV } & \multicolumn{2}{|r|}{ TBD } \\
\hline & & Obj. & $\mathrm{T}(\mathrm{s})$ & Obj. & $\mathrm{T}(\mathrm{s})$ & Obj. & $\mathrm{T}(\mathrm{s})$ \\
\hline $\begin{array}{llllllll}1 & 50 & 258 & 20 & 90 & 100 & 200 & 300\end{array}$ & 40 & 45 & 0.44 & 40 & 641.58 & 40 & 196.15 \\
\hline $2^{-} 44^{-} 510^{-} 20^{-} 90^{-} 0 \quad 100 \quad 200$ & 40 & 44 & 5.47 & 41 & 683.31 & 40 & 15.97 \\
\hline $3{ }_{-} 10 \overline{2} \_52 \overline{5} \_3 \overline{0}-9 \overline{0}-\overline{100} \overline{2} 00 \_300$ & 77 & 78 & 7.86 & 77 & 938.62 & 77 & 127.78 \\
\hline $4^{-} 113^{-} 647^{-} 20^{-} 90^{-} 100^{-} 200^{-} 300$ & 98 & 101 & 2.32 & 98 & 163.24 & 98 & 34.77 \\
\hline $5-77 \overline{7} 77, \overline{3} 0-\overline{9} 0 \overline{0}_{-} 10 \overline{0}-20 \overline{0}$ & 59 & 60 & 96.48 & 59 & $1,615.46$ & 59 & 12.98 \\
\hline $6^{-} \begin{array}{lllllll}13 \overline{5} & 77 \overline{7} & 2 \overline{0} & 9 \overline{0} & \overline{100} & \overline{2} 00 & 300\end{array}$ & 116 & 121 & 3.26 & 116 & $1,699.28$ & 116 & 663.7 \\
\hline $\begin{array}{llllllll}7 & 70 & \overline{7} 81 & \overline{2} 0 & \overline{9} 0 & \overline{0} & 10 \overline{0} & 20 \overline{0}\end{array}$ & 59 & 66 & 9.27 & 61 & 1,800 & 59 & 370.46 \\
\hline $8^{-} 88^{-}-102 \overline{2} \_2 \overline{0} \_9 \overline{0}-\overline{0}-10 \overline{0} \_200$ & 79 & 83 & 15.19 & 80 & 1,800 & 79 & 23.69 \\
\hline $\begin{array}{lllllll}9^{-} 12 \overline{5} & 130 \overline{8} & 2 \overline{0} & 9 \overline{0} & \overline{0} & 10 \overline{0} & 200\end{array}$ & 98 & 103 & 29.24 & 99 & 1,800 & 98 & 60.6 \\
\hline $1 \overline{0} \_15 \overline{3} \_157 \overline{\overline{7}} \_2 \overline{0} \_9 \overline{0}_{-} \overline{0}_{-} 10 \overline{0} \_200$ & 116 & 122 & 22.25 & 118 & 1,800 & 116 & 119.86 \\
\hline
\end{tabular}

\subsection{Automatic time-based $\mathrm{CMH}$}

Depending on instance structure, the automatic time-based algorithm selects either a fixed or blocking strategy. A brief analysis shows that the mean $(\mu)$ percentage difference between the minima and maxima of the active-task distribution graph over all the instances is 48.19 with standard deviation, 
$\sigma=29.82$. Therefore, the value $\mu-\sigma=18.37$ is used to filter instances that do not have an effective minima and therefore, $r=18.37 \%$ is selected. $d_{\text {min }}$ was set as 240, which corresponds to the smallest block size given by $\beta=6$ of the time-based decomposition. The variable strategy utilizes four configurations, $(\eta, 0,0),(\eta, 25,0),\left(\eta, 0, \eta^{\prime}\right)$ and $\left(\eta, 25, \eta^{\prime}\right)$, where $\eta^{\prime}$ corresponds to size of the following block. In this strategy, relaxed future adds the variables corresponding to an entire next block to the current block. This is to preserve the selected decomposition. The algorithm ends when an optimal solution is found or when all configurations have been tested. The fixed blocking strategy executes the configurations $(\beta, 80,0),(\beta, 90,0),(\beta, 80,50)$ and $(\beta, 90,50)$ in this order for all the block sizes (the same configuration utilized by the time-based decomposition). Tables 3,4 and 5 summarize the results of the automatic CMH algorithm on Data_100 and Data_137 instances.

The automatic time-based $\mathrm{CMH}$ solves 136 Data_137 instances and 96 Data_100 instances to optimality. Out of the four unsolved Data_100 instances, optimal solutions for two have not yet been established. The column $f$ indicates whether the fixed (0) or variable (1) blocking strategy has been implemented for the specific instance. Results demonstrate how a variable blocking strategy was utilized for all Data_100 instances, whereas a fixed strategy was adopted for almost all large Data_137 instances. The best results obtained by this $\mathrm{CMH}$ algorithm within the benchmark time limit of $1800 \mathrm{~s}$ are presented in aggregated Tables 6 and 7 . The best results obtained by other methods from the literature are also presented for comparison purposes. Table 8 provides a summary of the results obtained for the Data_10 instances along with the results obtained by other methods from the literature. KEB denotes Krishnamoorthy et al. (2012), SV denotes Smet and Vanden Berghe (2012), SWMV denotes Smet et al. (2014), Lin-Ying denotes Lin and Ying (2014), FL denotes Fages and Lapègue (2013), DMA denotes Baatar et al. (2015), NMA denotes Niraj Ramesh et al. (2018) and MH denotes Hojati (2018).

\section{Discussion of results}

Smet et al. (2014) and the automatic time based decomposition perform significantly better on the original Data_137 instances compared to other algorithms from the literature. For the Data_100 instances, Smet et al. (2014) produces best results followed by the automatic time-based $\mathrm{CMH}$. When it comes to the Data 10 instances, the time-based decomposition is, for the first time, capable of solving all Data_10 instances within the benchmark time limit of 1800 seconds. The automatic time-based $\mathrm{CMH}$, when utilizing the same parameter configuration for the whole set of benchmark instances also generates 10 optimal solutions, utilizing similar calculation times. In summary, the time-based decomposition and automatic time-based CMH has been able to produce optimal solutions for all the unsolved Data_10 instances. While there are competent techniques in the literature which are capable of producing equally good results over Data_100 or Data_137, the automatic time-based CMH proves 
Table 3: Results of automatic time-based $\mathrm{CMH}$ on the small and medium Data 137 instances. $f, \mathrm{LB}, \mathrm{Obj}$. and $\mathrm{T}(\mathrm{s})$ denote Fixed, lower bound, objective-value and algorithm runtime (seconds) respectively.

\begin{tabular}{|c|c|c|c|c|}
\hline Instance & $f$ & Obj. & $\mathrm{T}(\mathrm{s})$ & LB \\
\hline 1_23_40_66 & 1 & 20 & 0.02 & 20 \\
\hline $2^{-} 24^{-} 40^{-} 33$ & 1 & 20 & 0.02 & 20 \\
\hline $3^{-} 25^{-} 40^{-} 66$ & 1 & 20 & 0.02 & 20 \\
\hline $4^{-} 23^{-} 59^{-} 33$ & 1 & 20 & 0.17 & 20 \\
\hline $5-25-60-33$ & 1 & 20 & 0.13 & 20 \\
\hline 6_48_80-66 & 1 & 40 & 0.07 & 40 \\
\hline $7_{-}^{-} 51_{-}^{-} 80_{-}^{-} 66$ & 1 & 40 & 0.08 & 40 \\
\hline $8^{-} 48^{-} 85^{-} 33$ & 1 & 40 & 0.22 & 40 \\
\hline $9^{-} 49^{-} 10 \overline{4} \quad 33$ & 1 & 41 & 0.6 & 40 \\
\hline $\begin{array}{llll}1 \overline{0} & 5 \overline{1} & 11 \overline{1} & 66\end{array}$ & 1 & 40 & 0.11 & 40 \\
\hline $11^{-} 24-119-33$ & 1 & 20 & 0.27 & 20 \\
\hline $12 \_49 \_119 \_33$ & 1 & 40 & 0.33 & 40 \\
\hline $13^{-} 25^{-} 120^{-} 33$ & 1 & 20 & 0.86 & 20 \\
\hline $14-75-124-33$ & 1 & 60 & 0.43 & 60 \\
\hline $15^{-} 72^{-} 126^{-} 33$ & 1 & 60 & 0.42 & 60 \\
\hline $16^{-} 75^{-} 131^{-} 66$ & 1 & 60 & 0.25 & 60 \\
\hline $17^{-} 23-139-66$ & 1 & 20 & 0.66 & 20 \\
\hline 18_48_160-66 & 1 & 40 & 0.16 & 40 \\
\hline $19-97^{-} 160-33$ & 1 & 80 & 0.17 & 80 \\
\hline $20^{-} 99^{-} 163^{-} 33$ & 1 & 80 & 0.64 & 80 \\
\hline $21^{-} 93^{-} 175^{-} 33$ & 1 & 80 & 1.49 & 80 \\
\hline $22 \_47-180 \_66$ & 1 & 40 & 0.77 & 40 \\
\hline $23 \_74 \_180 \_66$ & 1 & 60 & 0.28 & 60 \\
\hline $24^{-} 11 \overline{0} \quad 20 \overline{0} \quad 33$ & 1 & 100 & 0.31 & 100 \\
\hline $25 \_120 \_200 \_33$ & 1 & 100 & 0.24 & 100 \\
\hline $26 \_116 \_203 \_66$ & 1 & 100 & 0.55 & 100 \\
\hline $27-49 \overline{2} 04 \_\overline{6} 6$ & 1 & 40 & 0.6 & 40 \\
\hline $28^{-} 75^{-}-208-66$ & 1 & 60 & 0.99 & 60 \\
\hline $2922 \quad 219-66$ & 1 & 20 & 2.48 & 20 \\
\hline $30 \_25 \_219-66$ & 1 & 20 & 1.35 & 20 \\
\hline $31^{-} 90^{-} 230^{-} 66$ & 1 & 80 & 0.63 & 80 \\
\hline $32-70 \_236-66$ & 1 & 60 & 2.32 & 60 \\
\hline $33-76 \_240 \_66$ & 1 & 60 & 0.43 & 60 \\
\hline $34^{-} 15 \overline{2} \quad 24 \overline{0} \quad 33$ & 1 & 120 & 0.44 & 120 \\
\hline $35-171-280-33$ & 1 & 140 & 0.66 & 140 \\
\hline $36 \_175 \_280 \_33$ & 1 & 140 & 0.69 & 140 \\
\hline $37^{-} 145^{-} 321^{-} 33$ & 0 & 120 & 12.26 & 120 \\
\hline 38_-147-347-66 & 0 & 120 & 5.22 & 120 \\
\hline $39^{-} 45 \overline{3} 51 \overline{6} 6$ & 1 & 40 & 2.49 & 40 \\
\hline $40^{-} 13 \overline{8} \quad 36 \overline{0} \quad 33$ & 1 & 120 & 0.71 & 120 \\
\hline $41^{-} 144^{-} 360^{-} 66$ & 1 & 120 & 1.76 & 120 \\
\hline $42^{-} 101^{-} 380^{-} 66$ & 1 & 80 & 2.68 & 80 \\
\hline $43^{-156} 387^{-66}$ & 0 & 140 & 6.1 & 140 \\
\hline $44 \_121 \_400 \_33$ & 1 & 100 & 0.7 & 100 \\
\hline $45 \_67 \_420 \_33$ & 0 & 60 & 2.04 & 60 \\
\hline $46^{-} 14 \overline{7} \quad 42 \overline{3} \quad 33$ & 1 & 120 & 4.05 & 120 \\
\hline $47_{-150 \_430 \_33}^{-150}$ & 1 & 120 & 4.91 & 120 \\
\hline $48 \_120 \_434 \_66$ & 1 & 100 & 20.37 & 100 \\
\hline 49_211_446_66 & 1 & 180 & 278.72 & 180 \\
\hline $50^{-} 187^{-} 447^{-} 66$ & 0 & 160 & 10.18 & 160 \\
\hline
\end{tabular}

\begin{tabular}{|c|c|c|c|c|}
\hline Instance & $f$ & Obj. & $\mathrm{T}(\mathrm{s})$ & LB \\
\hline $\begin{array}{llll}51 & 196 & 480 & 33\end{array}$ & 1 & 160 & 12.28 & 160 \\
\hline $52^{-} 205^{-} 480^{-} 66$ & 1 & 160 & 6.01 & 160 \\
\hline $53^{-} 127^{-} 487^{-} 66$ & 0 & 100 & 9.81 & 100 \\
\hline $54^{-} 175^{-} 492^{-} 66$ & 0 & 140 & 24.45 & 140 \\
\hline $55-85 \overline{4} 93 \quad \overline{6} 6$ & 1 & 70 & 12.13 & 70 \\
\hline $56^{-} 16 \overline{3} \quad 50 \overline{0} \quad 66$ & 0 & 140 & 11.13 & 140 \\
\hline $57^{-88} \overline{508} \overline{6} 6$ & 1 & 70 & 16.2 & 70 \\
\hline $58^{-} 15 \overline{8} \quad 51 \overline{7} \quad 66$ & 1 & 140 & 6.92 & 140 \\
\hline $59^{-} 70-\overline{5} 25-\overline{3} 3$ & 1 & 59 & 78.96 & 59 \\
\hline $60^{-} 18 \overline{1} \quad 54 \overline{9} \quad 66$ & 0 & 139 & 15.54 & 139 \\
\hline $61^{-} 121^{-} 557^{-} 66$ & 0 & 100 & 10.38 & 100 \\
\hline $62^{-} 101^{-} 571^{-} 33$ & 1 & 80 & 60.93 & 80 \\
\hline $63_{-}^{-} 97 \_\overline{5} 77_{-} \overline{6} 6$ & 1 & 80 & 5.12 & 80 \\
\hline $64^{-} 17 \overline{6} \quad 59 \overline{5} \quad 66$ & 1 & 160 & 9.69 & 160 \\
\hline $65^{-} 179^{-} 596^{-} 66$ & 0 & 159 & 16.34 & 159 \\
\hline $66^{-} 348^{-} 600^{-} 33$ & 1 & 300 & 6.06 & 300 \\
\hline $67^{-} 371^{-} 600^{-} 66$ & 1 & 300 & 13.42 & 300 \\
\hline $68^{-} 359^{-} 613^{-} 66$ & 1 & 300 & 15.7 & 300 \\
\hline $69^{-} 148^{-} 614^{-} 33$ & 0 & 120 & 13.03 & 120 \\
\hline $70^{-} 192^{-} 623^{-} 66$ & 1 & 160 & 3 & 160 \\
\hline $71^{-} 197^{-} 624^{-} 33$ & 0 & 8 & 38. & 158 \\
\hline $72^{-} 205^{-} 624^{-} 66$ & 1 & 0 & 9 & 160 \\
\hline $73^{-} 155^{-} 661^{-} 66$ & 0 & 120 & 42.16 & 120 \\
\hline $74^{-} 209^{-} 664^{-} 33$ & 0 & 180 & 8.56 & 180 \\
\hline $75^{-} 72 \overline{6} 65 \overline{3} 3$ & 1 & 60 & 128.88 & 60 \\
\hline $\begin{array}{llll}76^{-} & 16 \overline{2} & 68 \overline{3} & 66\end{array}$ & 0 & 140 & 22.45 & 140 \\
\hline $77^{-} 180^{-} 688^{-} 33$ & 1 & 160 & 43 & 160 \\
\hline $78^{-} 199^{-} 688^{-} 66$ & 1 & 160 & 11.52 & 160 \\
\hline $79^{-} 94 \quad \overline{6} 89 \overline{3} 3$ & 0 & 80 & 6.9 & 80 \\
\hline $80^{-} 11 \overline{2} \quad 69 \overline{1} \quad 33$ & 0 & 99 & 16.25 & 99 \\
\hline $81^{-} 97 \overline{6} 92 \quad \overline{6} 6$ & 1 & 80 & 38.15 & 80 \\
\hline $82-89-697-66$ & 1 & 80 & 2 & 80 \\
\hline $83^{-} 22 \overline{2} \quad 70 \overline{0} \quad 66$ & 0 & 180 & 31.3 & 180 \\
\hline 84-136-718_66 & 1 & 120 & 479.85 & 120 \\
\hline $85^{-} 217^{-} 720^{-} 66$ & 1 & 180 & 11.39 & 180 \\
\hline $86^{-} 178^{-} 721^{-} 33$ & 0 & 140 & 20.26 & 140 \\
\hline $87^{-} 203^{-} 735^{-} 33$ & 1 & 170 & 341.31 & 170 \\
\hline $88^{-} 137^{-} 777^{-} 66$ & 0 & 120 & 22.55 & 120 \\
\hline $89^{-} 88 \overline{7} 88 \overline{3} 3$ & 1 & 70 & 47.53 & 70 \\
\hline $90^{-} 15 \overline{7} \quad 79 \overline{1} \quad 66$ & 0 & 139 & 27.06 & 139 \\
\hline 91_147_851_66 & 0 & 118 & 85.27 & 118 \\
\hline $92^{-} 126^{-} 856^{-} 66$ & 0 & 98 & 35.52 & 98 \\
\hline $93^{-} 141^{-} 856^{-} 66$ & 0 & 119 & 30.53 & 119 \\
\hline $94^{-} 93 \overline{8} 81 \quad \overline{3} 3$ & 1 & 80 & 102.76 & 80 \\
\hline $95^{-} 20 \overline{4} \quad 88 \overline{2} \quad 33$ & 0 & 170 & 15.54 & 170 \\
\hline $96-98 \quad \overline{8} 86 \quad \overline{6} 6$ & 1 & 80 & 50.75 & 80 \\
\hline $97^{-} 38 \overline{3} \quad 89 \overline{5} \quad 33$ & 1 & 300 & 38.3 & 300 \\
\hline $98^{-} 91 \quad \overline{8} 96 \quad \overline{3} 3$ & 0 & 80 & 14.37 & 80 \\
\hline $99^{-} 17 \overline{6} \quad 95 \overline{6} \quad 66$ & 1 & 160 & 61.24 & 160 \\
\hline $\begin{array}{llll}10 \overline{0} & 19 \overline{4} & 95 \overline{6} & 66\end{array}$ & 1 & 160 & $1,068.23$ & 160 \\
\hline
\end{tabular}

to be an efficient strategy which is capable of generating high quality solutions over all datasets. More specifically, this strategy performs well even on very large instances and therefore can be adapted for solving real-world scenarios.

The merit of the automatic-CMH lies in the fact that it is a simple constructive heuristic that efficiently generates very high quality solutions (optimal solutions for 242 out of 247 instances) in a single pass of the algorithm. 
Table 4: Results of automatic time-based $\mathrm{CMH}$ on the large Data 137 instances. $f$, LB Obj. and T(s) denote Fixed, lower bound, objective-value and algorithm runtime (seconds) respectively.

\begin{tabular}{|c|c|c|c|c|}
\hline Instance & $f$ & Obj. & $\mathrm{T}(\mathrm{s})$ & LB \\
\hline 101_166_997_66 & 0 & 140 & 51.92 & 140 \\
\hline $102^{-} 179^{-} 997^{-} 66$ & 0 & 138 & 57.65 & 138 \\
\hline $103^{-} 348^{-} 102 \overline{4} 33$ & 0 & 300 & 347.05 & 300 \\
\hline $104^{-} 181^{-} 1057^{-} 33$ & 0 & 146 & 25.02 & 146 \\
\hline $105^{-} 173_{-}^{-} 1075_{-}^{-} 66$ & 0 & 50 & 77.31 & 150 \\
\hline 106 $121_{-}^{-} 1096_{-}^{-} 33$ & 0 & 100 & 25.78 & 100 \\
\hline $107 \_114 \_1112 \_33$ & 1 & 100 & 69.79 & 100 \\
\hline $108^{-} 162^{-} 1115^{-} 33$ & 0 & 128 & 29.36 & 128 \\
\hline 109_205_1115_33 & 0 & 157 & 29.95 & 157 \\
\hline $110^{-} 183^{-} 1143^{-} 66$ & 0 & 155 & 82.42 & 155 \\
\hline 111 155_1211_33 & 0 & 139 & 33.29 & 139 \\
\hline 112_200_1213_33 & 0 & 169 & 32.02 & 169 \\
\hline $113^{-} 141^{-} 1221^{-} 66$ & 0 & 110 & 83.43 & 110 \\
\hline $114 \_157 \_1227 \_33$ & 0 & 138 & 36.49 & 138 \\
\hline 115_228_1257_33 & 0 & 177 & 43.97 & 177 \\
\hline $116 \_205 \_1262 \_66$ & 0 & 176 & 105.18 & 176 \\
\hline $117^{-} 192-1285^{-}-33$ & 0 & 149 & 47.04 & 149 \\
\hline $118^{-} 180^{-} 1302^{-} 33$ & 0 & 147 & 46.04 & 147 \\
\hline $119-236 \_1335-33$ & 0 & 188 & 47.9 & 188 \\
\hline
\end{tabular}

\begin{tabular}{|c|c|c|c|c|}
\hline Instance & $f$ & Obj. & $\mathrm{T}(\mathrm{s})$ & LB \\
\hline $\begin{array}{llll}120 & 228 & 1341 & 33\end{array}$ & 0 & 37 & 47. & 187 \\
\hline $121^{-} 147^{-} 1345^{-} 33$ & ( & 20 & 0. & 120 \\
\hline $122^{-} 422^{-}-1358^{-} 66$ & 0 & 8 & 235.6 & 348 \\
\hline $123^{-187} 1376-33$ & 0 & 59 & 45.2 & 159 \\
\hline 124_198_1383_33 & 0 & 158 & 55. & 158 \\
\hline $125 \_157 \_1448 \_33$ & & 130 & 208. & 130 \\
\hline $126^{-} 193$ & & 7 & 60. & 167 \\
\hline $127^{-} 192$ & & 0 & 72. & 170 \\
\hline $128^{-207^{-}} 15$ & & 178 & 181 & 178 \\
\hline 129-233-1546_33 & & 178 & 79 & 178 \\
\hline $130^{-} 176^{-} 1562^{-} 66$ & & 14 & 433. & 140 \\
\hline $131^{-} 415^{-1610^{-}} 33$ & & 348 & $95 .($ & 348 \\
\hline $132^{-} 216^{-} 1645^{-} 33$ & & 186 & 266. & 186 \\
\hline $133^{-211^{-}} 1647^{-} 33$ & & 18 & 294. & 185 \\
\hline 134 184_1776_66 & & 16 & 239 & 160 \\
\hline $135 \_213 \_1988 \_33$ & 0 & 179 & 153.74 & 179 \\
\hline 136_216_2000_66 & 0 & 179 & 431.04 & 179 \\
\hline $137_{-} 245^{-} 2105_{-}-33$ & 0 & 190 & 206.14 & 190 \\
\hline
\end{tabular}

The key feature of the automatic-CMH which makes it efficient at solving large and difficult instances is its underlying decomposition strategy. The employeebased decomposition applied by Smet et al. (2014) requires employing larger block sizes in order to improve solution quality, which in turn affects the algorithm run time. The advantage of the time-based decomposition lies in its ability to utilize much smaller blocks and yet still generate high quality solutions when combined with sufficient overlap and relaxed future, the presence of which impacts runtime far less than increasing block size. Experiments show, however, that implementing overlap in a employee-based decomposition does not significantly improve the solution quality. In order to be able to implement overlap or relaxed future, it requires identifying an appropriate order of solving subproblems. This is to ensure that the compromise in local optimality of subproblems when employing overlap or relaxed future reflects in the final solution quality improvement for the entire problem. In this problem, timebased decomposition imposes a natural ordering for the subproblems which enables overlap and relaxed future features to be effectively implemented.

\section{Conclusions and future work}

The present paper compared two different decomposition strategies applied within a constructive matheuristic framework when applied to the shift minimization personnel task scheduling problem (SMPTSP). The paper also studied the features of the decomposition strategy which makes it suitable for implementing key CMH design parameters such as overlap and relaxed future. Not only do these features improve solution quality, but they also play a pivotal role in improving the scalability of the algorithm, which makes it suitable for 
Table 5: Results of automatic time-based CMH on the Data 100 instances. $f$, LB, Obj. and $\mathrm{T}(\mathrm{s})$ denote Fixed, lower bound, objective-value and algorithm runtime (seconds) respectively.

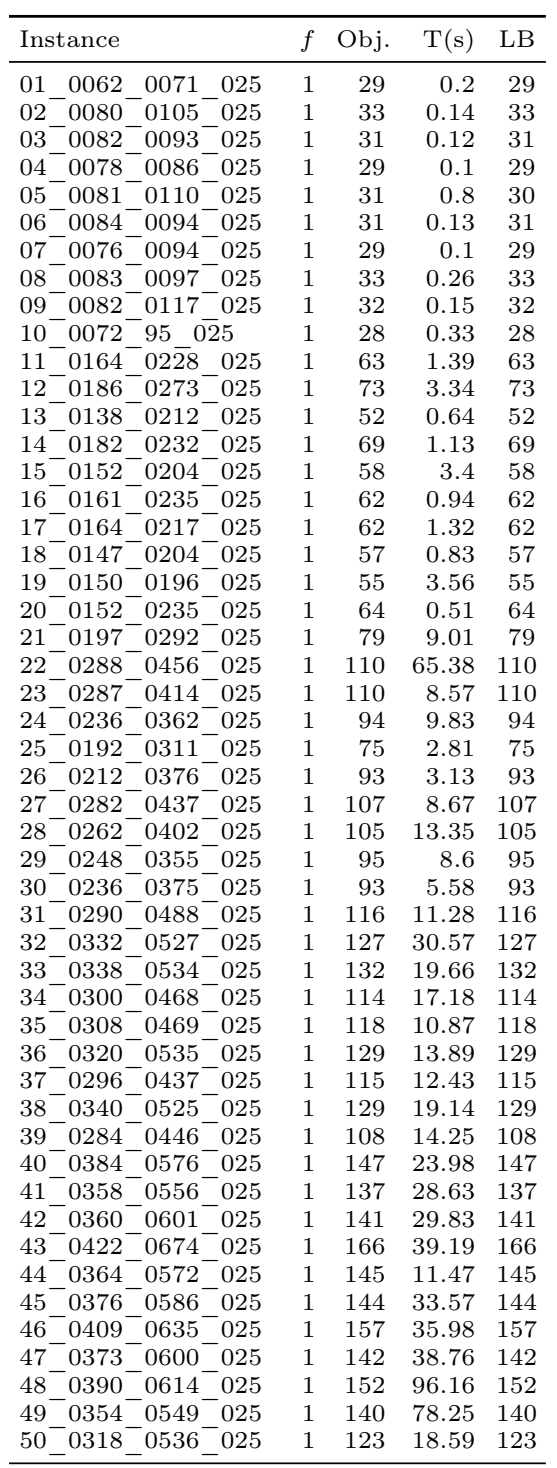

\begin{tabular}{|c|c|c|c|c|}
\hline Instance & $f$ & Obj. & $\mathrm{T}(\mathrm{s})$ & $\mathrm{LB}$ \\
\hline $51 \quad 0514 \quad 0832 \quad 025$ & 1 & 197 & 123.3 & 197 \\
\hline 52_0448_0767_025 & 1 & 171 & 70.43 & 171 \\
\hline 53_0486_0746_025 & 1 & 186 & 56.31 & 186 \\
\hline 54_0498_0850_025 & 1 & 190 & 71.66 & 190 \\
\hline 55_0524_0920_025 & 1 & 201 & 182.43 & 201 \\
\hline $56^{-} 0538^{-} 0911^{-} 025$ & 1 & 206 & 151.7 & 206 \\
\hline $57^{-} 0440^{-} 0737^{-} 025$ & 1 & 169 & 43.15 & 169 \\
\hline $58-0348-0562-025$ & 1 & 132 & 28.84 & 132 \\
\hline $59-0460 \quad 0689025$ & 1 & 176 & 63.72 & 176 \\
\hline 60_0443_0783_025 & 1 & 173 & 67.91 & 173 \\
\hline $61^{-} 0551^{-} 0891^{-} 025$ & 1 & 222 & 152.63 & 222 \\
\hline $62-0610-1096-025$ & 1 & 262 & 254.33 & 262 \\
\hline $63^{-} 0524^{-} 0905^{-} 025$ & 1 & 203 & 117.44 & 203 \\
\hline $6403660570-025$ & 1 & 140 & 25.67 & 140 \\
\hline 65_0456_0764_025 & 1 & 179 & 81.26 & 179 \\
\hline 66_0492_0775_025 & 1 & 189 & 99.23 & 189 \\
\hline $670597-0949025$ & 1 & 230 & 254.18 & 230 \\
\hline 68_0561_0958_025 & 1 & 219 & 79.54 & 219 \\
\hline $69^{-} 0550^{-} 0891^{-} 025$ & 1 & 211 & 114.42 & 211 \\
\hline 70_0550_0990_025 & 1 & 211 & 02.18 & 211 \\
\hline 71_0528_0895_025 & 1 & 202 & 193.27 & 202 \\
\hline $72^{-} 0604^{-} 0997^{-} 025$ & 1 & 230 & 543.07 & 230 \\
\hline 73_0604_0999_025 & 1 & 239 & 262.39 & 239 \\
\hline $74^{-} 0563-0941-025$ & 1 & 217 & 157.56 & 217 \\
\hline 75_0518_0870_025 & 1 & 204 & 217.5 & 204 \\
\hline 76_0642_1107_025 & 1 & 246 & 343.58 & 246 \\
\hline 77_0648_1123_025 & 1 & 249 & 1,800 & 248 \\
\hline 78_0548_0941_025 & 1 & 210 & 100.11 & 210 \\
\hline 79_0638_1052_025 & 1 & 246 & 172.93 & 246 \\
\hline $80^{-} 0578^{-} 0885^{-} 025$ & 1 & 222 & 165.53 & 222 \\
\hline 81_0647_1181-025 & 1 & 265 & 154.37 & 265 \\
\hline $82^{-} 0734^{-} 1265^{-} 025$ & 1 & 289 & 148.21 & 289 \\
\hline $83^{-} 0551^{-} 0941^{-} 025$ & 1 & 206 & 110.42 & 206 \\
\hline $84^{-} 0644^{-} 1121^{-} 025$ & 1 & 247 & 457.87 & 247 \\
\hline $85^{-} 0688^{-} 1111^{-} 025$ & 1 & 263 & 117.84 & 263 \\
\hline $86-0628-1136-025$ & 1 & 241 & 251.61 & 241 \\
\hline 87_0765_1323_025 & 1 & 288 & 237.64 & 288 \\
\hline 88_0808_1346_025 & 1 & 310 & $1,396.74$ & 310 \\
\hline 89_0790_1371_025 & 1 & 320 & $1,677.57$ & 319 \\
\hline 90_0810_1443_025 & 1 & 312 & 651.57 & 312 \\
\hline $910754-1287^{-025}$ & 1 & 289 & 330.8 & 289 \\
\hline 92_0948_1583_025 & 1 & 364 & 722.84 & 364 \\
\hline $93^{-} 0820^{-} 1478^{-} 025$ & 1 & 340 & 247.61 & 340 \\
\hline 94_0812_1394_025 & 1 & 318 & 1,802 & 313 \\
\hline $95^{-} 0696^{-} 1162^{-} 025$ & 1 & 266 & 190.09 & 266 \\
\hline $96^{-} 0710^{-} 1250^{-} 025$ & 1 & 272 & 601.47 & 272 \\
\hline $97 \_0886 \_1605 \_025$ & 1 & 340 & 691.2 & 340 \\
\hline 98_0750_1334_025 & 1 & 289 & 295.72 & 289 \\
\hline 99_0564_0955_025 & 1 & 221 & 311.81 & 221 \\
\hline $10 \overline{0} \quad 080 \overline{6} \quad 137 \overline{4} \quad 025$ & 1 & 310 & 491.27 & 310 \\
\hline
\end{tabular}

application in large real-world scenarios. The results of an extensive computational study conducted shows that the resulting automated CMH strategy is capable of producing optimal solutions for a large number of benchmark instances. More specifically, this strategy has been able to solve all Data 10 instances to optimality for the first time. Another major contribution of the 
Table 6: Results of automatic time-based CMH on the Data_137 instances.

\begin{tabular}{lrrrrrrr}
\hline & KEB & SV & SWMV & $\begin{array}{r}\text { Lin- } \\
\text { Ying }\end{array}$ & FL & MH & $\begin{array}{r}\text { auto- } \\
\text { CMH }\end{array}$ \\
\hline Number of optimal solutions & 67 & 81 & 137 & 105 & 137 & 129 & 136 \\
Average solution & 127 & 123.04 & 122.24 & 122.72 & 122.24 & 122.48 & 122.25 \\
Average calculation time(s) & 544.3 & 958.91 & 55.52 & 637.5 & - & 15.3 & 59.54 \\
Maximum calculation time(s) & 1800 & 1800 & 604.87 & 1800 & - & 1690.9 & 1068.23 \\
\hline
\end{tabular}

Table 7: Results of automatic time-based CMH on the Data 100 instances

\begin{tabular}{lrrrc}
\hline & FL & MH & SWMV & auto-CMH \\
\hline Number of optimal solutions & 46 & 84 & 98 & 96 \\
Average solution & 166.2 & 164.56 & 164.4 & 164.46 \\
Average calculation time(s) & - & 154.5 & 88.46 & 179.21 \\
Maximum calculation time(s) & 1800 & 1800 & 683.07 & 1,800 \\
\hline
\end{tabular}

Table 8: Results of automatic time-based CMH on the Data 10 instances. $f$, Obj. and T(s) denote Fixed, objective-value and algorithm runtime (seconds) respectively.

\begin{tabular}{|c|c|c|c|c|c|c|c|c|c|}
\hline \multirow[b]{2}{*}{ Instance } & \multicolumn{3}{|c|}{ auto-CMH } & \multicolumn{2}{|r|}{ SWMV } & \multicolumn{2}{|c|}{ DMA } & \multicolumn{2}{|r|}{ NMA } \\
\hline & $f$ & Obj. & $\mathrm{T}(\mathrm{s})$ & Obj. & $\mathrm{T}(\mathrm{s})$ & Obj. & $\mathrm{T}(\mathrm{s})$ & Obj. & $\mathrm{T}(\mathrm{s})$ \\
\hline $\begin{array}{llllllll}1 & 50 & 258 & 20 & 90 & 100 & 200 & 300\end{array}$ & 1 & 40 & 9.42 & 40 & 641.58 & 41 & 1,800 & 40 & 33.8 \\
\hline $2^{-} 44^{-} 510^{-} 20_{-}^{-} 90^{-} 0 \_100 \_200$ & 1 & 40 & 176.52 & 41 & 683.31 & 40 & 61 & 40 & 3007 \\
\hline 3 ${ }_{-}^{-} 10 \overline{2} \_52 \overline{5} \_3 \overline{0} \_9 \overline{0} \_\overline{100} \_\overline{200} 3300$ & 0 & 77 & 128.75 & 77 & 938.62 & 77 & 198 & 77 & 591.3 \\
\hline 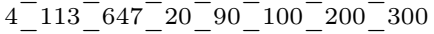 & 0 & 98 & 33.41 & 98 & 163.24 & 98 & 324 & 98 & 299.7 \\
\hline $5^{-} 77 \overline{7} 77, \overline{3} 0 \overline{9} 0 \overline{0} \quad 10 \overline{0} \quad 20 \overline{0}$ & 0 & 59 & 12.76 & 59 & $1,615.46$ & 59 & 495 & - & - \\
\hline $6{ }_{-}^{-} 13 \overline{5} \_77 \overline{7} \_2 \overline{0} \_9 \overline{0} \_\overline{100} \_\overline{200} \_300$ & 0 & 116 & 664.78 & 116 & $1,699.28$ & 121 & 1,801 & 116 & 3691.8 \\
\hline $7_{-}^{-} 70 \_\overline{7} 81 \_\overline{2} 0 \_\overline{9} 0 \_\overline{0} \_10 \overline{00} \_20 \overline{0}$ & 0 & 59 & 371.83 & 61 & 1,800 & 59 & 147 & - & - \\
\hline $\begin{array}{lllllll}8^{-} 88^{-} & 102 \overline{2} & 2 \overline{0} & 9 \overline{0} & \overline{0} & 10 \overline{0} & 200\end{array}$ & 0 & 79 & 23.54 & 80 & 1,800 & 79 & 1,286 & - & - \\
\hline $9^{-} 12 \overline{5} 130 \overline{8} \_2 \overline{0}-9 \overline{0} \_\overline{0} 10 \overline{0}-200$ & 0 & 98 & 60.99 & 99 & 1,800 & 106 & 1,801 & - & - \\
\hline 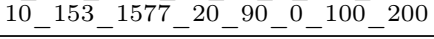 & 0 & 116 & 119.22 & 118 & 1,800 & 128 & 1,806 & - & - \\
\hline nal solutions & & & 10 & & 6 & & 0 & & 5 \\
\hline Average solution & & & 78.2 & & 78.9 & & 80.8 & & - \\
\hline Average calculation time(s) & & & 160.12 & & $1,294.15$ & & 971.9 & & - \\
\hline Maximum calculation time(s) & & & 664.78 & & 1,800 & & 1,800 & & 5000 \\
\hline
\end{tabular}

paper is the development of a $\mathrm{CMH}$ strategy which automatically generates a suitable time-based decomposition based on instance structure.

For the SMPTSP, the time-based decomposition proves to be beneficial. Future research can be aimed at identifying problem features on which decomposition can be based such that the resulting order of solving subproblems is suitable for implementing an effective $\mathrm{CMH}$ strategy and the design parameters discussed. It is also worth identifying problem classes or problem features which makes a time or time-like feature based decomposition suitable.

Acknowledgements We would like to thank Luke Conolly (KU Leuven) for providing editorial consultation. 


\section{References}

D. Baatar, M. Krishnamoorthy, and A. T. Ernst. A triplet-based exact method for the shift minimisation personnel task scheduling problem. In N. Bansal and I. Finocchi, editors, Algorithms - ESA 2015, pages 59-70. Springer Berlin Heidelberg, 2015.

R. C. Chandrasekharan, T. A. M. Toffolo, and T. Wauters. Analysis of a constructive matheuristic for the traveling umpire problem. Journal of Quantitative Analysis in Sports, 15(1):41-57, 2019.

F. Della Croce, A. Grosso, and F. Salassa. A matheuristic approach for the two-machine total completion time flow shop problem. Annals of Operations Research, 213:67-78, 2014.

J. V. den Bergh, J. Belién, P. D. Bruecker, E. Demeulemeester, and L. D. Boeck. Personnel scheduling: A literature review. European Journal of Operational Research, 226(3):367 - 385, 2013.

D. Dowling, M. Krishnamoorthy, H. Mackenzie, and D. Sier. Staff rostering at a large international airport. Annals of Operations Research, 72:125-147, 1997.

J. Fages and T. Lapègue. Filtering atmostnvalue with difference constraints: Application to the shift minimisation personnel task scheduling problem. In C. Schulte, editor, Principles and Practice of Constraint Programming, pages 63-79. Springer Berlin Heidelberg, 2013.

M. Fischetti and A. Lodi. Local branching. Mathematical Programming, 98: 23-47, 2003.

M. Hojati. A greedy heuristic for shift minimization personnel task scheduling problem. Computers and Operations Research, 100:66-76, 2018.

A. W. Kolen, J. K. Lenstra, C. H. Papadimitriou, and F. C. Spieksma. Interval scheduling: A survey. Naval Research Logistics (NRL), 54(5):530-543, 2007.

M. Krishnamoorthy, A. Ernst, and D. Baatar. Algorithms for large scale shift minimisation personnel task scheduling problems. European Journal of Operational Research, 219:34-48, 2012.

S. Lin and K. Ying. Minimizing shifts for personnel task scheduling problems: A three-phase algorithm. European Journal of Operational Research, 237: 323-334, 2014.

V. Maniezzo, T. Stützle, and S. Voß. Matheuristics: Hybridizing Metaheuristics and Mathematical Programming. Springer Publishing Company, Incorporated, 1st edition, 2009. ISBN 144191305X, 9781441913050.

D. Niraj Ramesh, M. Krishnamoorthy, and A. T. Ernst. Efficient models, formulations and algorithms for some variants of fixed interval scheduling problems. In R. Sarker, H. A. Abbass, S. Dunstall, P. Kilby, R. Davis, and L. Young, editors, Data and Decision Sciences in Action, pages 43-69, Cham, 2018. Springer International Publishing.

H. G. Santos, T. A. M. Toffolo, R. A. M. Gomes, and S. Ribas. Integer programming techniques for the nurse rostering problem. Annals of Operations Research, 239:225-251, 2016. 
P. Smet and G. Vanden Berghe. A matheuristic approach to the shift minimisation personnel task scheduling problem. Proceedings of the 9th international conference on the practice and theory of automated timetabling, PATAT, 2012, pages 145-151, 2012.

P. Smet, T. Wauters, M. Mihaylov, and G. V. Berghe. The shift minimisation personnel task scheduling problem: A new hybrid approach and computational insights. Omega, 46:64 - 73, 2014.

O. Solyali. The shift minimization personnel task scheduling problem: An effective lower bounding procedure. Hacettepe Úniversitesi İktisadi ve İdari Bilimler Fakúltesi Dergisi, 34(2), 2016.

L. A. Wolsey. Integer programming. Wiley-interscience series in discrete mathematics and optimization. Wiley, New York (N.Y.), 1998. ISBN 0471283665. 